\title{
Determinantes socioeconómicos en la estimación de la disponibilidad a pagar del proyecto de agua potable y saneamiento en el centro poblado de Paxa, distrito de Tiquillaca - Puno 2017
}

\section{Socioeconomic determinants in the estimation of the willingness to pay of the potable water and sanitation project in the town of Paxa, district of Tiquillaca - Puno 2017}

\author{
${ }^{1}$ Universidad Nacional del Altiplano de Puno, e-mail: eliascahui95@gmail.com \\ ${ }^{2}$ Universidad Nacional del Altiplano de Puno, e-mail: itudela@unap.edu.pe \\ ${ }^{3}$ Universidad Nacional del Altiplano de Puno, e-mail: ahuamani22@hotmail.com
}

Elias Cahui-Cahui ${ }^{1}$, Juan Walter Tudela-Mamani ${ }^{2}$, Alcides Huamaní-Peralta ${ }^{3}$

\author{
Recibido e1: 19/12/2018 \\ Aceptado e1: 27/03/2019
}

\section{RESUMEN}

El objetivo del estudio fue estimar la disponibilidad a pagar (DAP) media de las familias del centro poblado de Paxa para la sostenibilidad del proyecto del servicio de agua potable y saneamiento rural a través del método de valoración contingente(MVC) con formatos de preguntas tipo referéndum y doble limite. La información fue recolectada a través de una encuesta aplicada a una muestra de 112 jefes de familia. Se estimó una DAP media de S/ 3.22 mes/familia, la DAP fue incidida por variables socioeconómicas como el precio hipotético, ingreso familiar, edad y educación. Asimismo mediante el análisis correlacional de Pearson se determinó una relación directa y positiva entre la DAP y la capacidad de pago (CP). Los resultados permitirán a los tomadores de decisiones generar el planteamiento de políticas que permitan garantizar la sostenibilidad del proyecto.

Palabras clave: Valoración contingente, disponibilidad a pagar, agua potable y saneamiento, formato referéndum y doble límite, sostenibilidad de proyectos.

\section{ABSTRAGT}

The objective of the study is to estimate the average willingness to pay (WTP) of the families in the town of Paxa for the sustainability of the potable water and rural sanitation service project through the contingent valuation method (CVM) with question type formats referendum and double limit. The information was collected through a $\mathbf{8}$ survey applied to a sample of 112 heads of family. An average DAP of S / 3.22 month / family was estimated; the DAP was affected by socioeconomic variables such as the hypothetical price, family income, age and education. Likewise, Pearson's correlational analysis determined a direct and positive relationship between the WTP and the payment capacity (PC). The results will allow decision makers to generate the policy approach that will guarantee the sustainability of the project.

Keywords: Contingent valuation, willingness to pay, potable water and sanitation, referendum format and double bound, sustainability of projects. 


\section{INTRODUCCIÓN}

El agua y el saneamiento son elementos esenciales del desarrollo sostenible, que junto a la higiene adecuada, constituyen pilares de la salud humana y el bienestar (UN-Water, 2016). Para la OMS (2017) mejorar el suministro de agua, del saneamiento y de la gestión de los recursos hídricos impulsa no solo el crecimiento económico de un país sino también a la reducción de la pobreza. Sin embargo, a nivel mundial alrededor de 3 de cada 10 personas carecen de acceso al servicio de agua potable en el hogar, y 6 de cada 10 personas de un saneamiento seguro; como consecuencia de un rápido crecimiento demográfico (OMS \& UNICEF, 2017). Las poblaciones con mayor índice de pobreza y menores índice de salud y educación, principalmente las zonas rurales, son los más perjudicados, por sus altos costos de atención, dejando vulnerables a la población infantil quienes contraen enfermedades gastrointestinales, nutricionales y de trastornos neonatales cobrando más de 1.5 millones de vidas de niños menores de 5 años cada año (UNICEF, 2007).

En el Perú aún coexiste una gran brecha respecto a la cobertura y calidad de los servicios de agua potable y saneamiento básico que se dotan en el área urbana respecto al área rural, Según el INEI 82 (2018) al año 2017, de la población que accedió al agua por red pública, $94.4 \%$ correspondió al área urbana mientras que $72.2 \%$ al área rural, asimismo con respecto al saneamiento básico $88.7 \%$ de la población urbana accedió a un servicio por red de alcantarillado con respecto al $17.5 \%$ de la población rural, por lo que es necesario que los esfuerzos del país orientados hacia las zonas rurales sean incrementados significativamente.

Nuestro país dispone de herramientas adecuadas que permiten la elaboración de proyectos de agua potable y saneamiento en el ámbito rural; como para su ejecución, sin embargo aún existe falencias en actividades que permitan una adecuada administración, operación y mantenimiento, con la finalidad de prevenir su deterioro y garantizar su sostenibilidad, durante el tiempo para el cual fueron diseñados, ademas Parra, Vargas, \& Castellar (2005) señalan "aunque las inversiones sean cuantiosas, dichos proyectos no lograran los impactos positivos esperados en la calidad de vida de las comunidades, dada su baja sostenibilidad de los mismos", siendo la causa principal del fracaso, la falta de estudios de posibilidades de pago por parte de los beneficiarios para garantizar la sostenibilidad reflejado en los costos de operación y mantenimiento de los proyectos (Gutierrez, 2015).

Un gran reto es lograr que los servicios de agua potable y saneamiento derivados de proyectos de inversión pública sean realmente sostenibles y para ello, son necesarios estudios que permitan garantizar dicha sostenibilidad (costos de operación y mantenimiento) así como acciones en educación sanitaria y capacitación para la población.

Existe una vasta cantidad de antecedentes en la literatura internacional de investigaciones que estiman la DAP a través del método de valoración contingente (MVC) por la mejoría de los servicios básicos como son el agua y saneamiento, pero su aplicación en los países en desarrollo para Wang, Shi, Kim, \& Kamata (2013) continúa siendo limitada. Autores como Jianjun, Wenyu, Ying, \& Wang (2016); Kebede \& Tariku (2016) aportan algunas pautas de estudios internacionales que se han realizado haciendo uso del MVC para mejoras del servicio de agua. De igual forma destacan Tudela, Leos, \& Zavala (2018) quienes hacen hincapié del tema en el ámbito nacional. En todos los casos, el MVC permitió obtener la valoración económica del servicio propuesto, teniendo como punto de partida el planteamiento de una mejora en las condiciones de vida de los beneficiarios (Tudela, 2017).

El objetivo de esta investigación es estimar la disponibilidad a pagar (DAP) media de las familias del centro poblado de Paxa para la sostenibilidad del proyecto de agua potable y saneamiento siguiendo la línea del método de valoración contingente (MVC) con formatos tipo referéndum y doble limite, este último como aporte a la literatura local y la muestra de evidencia empírica 
de sus bondades frente al primero, cuyos datos se analizaron mediante un modelo Logit. Asimismo los objetivos específicos planteados son: analizar los principales factores socioeconómicos que inciden en la disponibilidad a pagar (DAP) y determinar la relación entre la capacidad de pago (CP) y la disponibilidad a pagar (DAP) de las familias del centro poblado de Paxa.

\section{MARCO TEÓRICO}

\section{Método de Valoración Contingente}

El MVC consiste en simular por medio de encuestas y escenarios hipotéticos un mercado para un bien o servicio para los que no existe mercado (Mendieta, 2005). El MVC desde el enfoque de la diferencia de la función indirecta de utilidad propuesto por Hanemann, asume que un individuo calcula su DAP y las compara con el pago ofrecido en la encuesta en función a su ingreso (Vásquez, Cerda, \& Orrego, 2007), el cual tiene la siguiente representación:

$$
u_{i}=v_{i}\left(p, y ; q_{i}\right)
$$

Donde $i=0$ es la situación inicial e $i=1$ es la situación modificada (mejora los servicios de agua y saneamiento), es un vector de precios que enfrentan los individuos por sus bienes, $y$ representa el ingreso familiar y $q_{i}$ constituye a un vector de características socioeconómicas observables de los individuos.

El supuesto principal del MVC es que la función de utilidad tiene componentes desconocidos para el investigador, lo cual sirve para generar una estructura estocástica de la función de utilidad representada por la ecuación (1). De esta forma la función de utilidad indirecta puede expresarse como:

$$
u_{i}=v_{i}\left(p, y ; q_{i}\right)+\varepsilon_{i}
$$

Donde $\varepsilon_{i}$ es el error estocástico con media cero. La ecuación (2) representa la función de utilidad determinística para el individuo, el mismo que se utiliza para analizar y describir las medidas de cambio en el bienestar. El MVC enfrenta al individuo a una elección entre una mejora (servicios de agua y saneamiento, y así garantizar la sostenibilidad del proyecto) de $q_{0}$ a $q_{1}$, por la cual se debe pagar una cantidad $A_{t}$ (suma de dinero propuesto), o no tener la mejora y no pagar. Sin embargo, la verdadera valoración expresada en el monto a pagar por el individuo no es observable, y lo único que es factible saber a partir de la respuesta de los individuos es si ésta es mayor o menor que la cantidad ofrecida $A_{t}$. Por lo tanto, la probabilidad de una respuesta positiva por parte del individuo está dado por la siguiente expresión:

$$
\begin{aligned}
\operatorname{Pr}(s i) & =\operatorname{Pr}\left[v_{1}\left(p, y-A_{i} ; q_{1}\right)+\varepsilon_{1}>v_{0}\left(p, y ; q_{0}\right)+\varepsilon_{0}\right] \\
& =\operatorname{Pr}\left[v_{1}\left(p, y-A_{i} q_{1}\right)-v_{0}\left(p, y ; q_{0}\right)>\varepsilon_{1}-\varepsilon_{0}\right] \\
& =\operatorname{Pr}\left[\Delta v>\varepsilon_{0}-\varepsilon_{1}\right] \\
& =\operatorname{Pr}[\Delta v>n] \\
& =F n[\Delta v]
\end{aligned}
$$

En la ecuación (3), es la función de distribución acumulada de $n$ y $n=\varepsilon_{0}-\varepsilon_{1}$. Si se asume que en la ecuación (3) los errores son ruido blanco, entonces se tiene:

$$
\Delta v=v_{1}\left(p, y-A_{i} ; q_{1}\right)-v_{0}\left(p, y ; q_{0}\right)
$$

$\mathrm{Si}$ suponemos una forma funcional lineal dependiendo del ingreso de la forma $v_{j}=a_{j}+\beta y$, donde $\beta$ representa la utilidad marginal del ingreso, y se le reemplaza en la ecuación (26) obtenemos:

$$
\begin{aligned}
\Delta v & =a_{1}+\beta\left(y-A_{t}\right)-\left(a_{0}+\beta y\right) \\
& =a_{1}+\beta y-\beta A_{t}-a_{0}-\beta y \\
& =\left(a_{1}-a_{0}\right)-\beta A_{t} \\
& =a-\beta A_{t}
\end{aligned}
$$

Donde $\beta>0$, dado que el valor esperado de la utilidad $(v)$ se incrementa con el ingreso, lo que implica que mientras mayor sea el valor de menor será la , por lo tanto, la probabilidad de que un individuo responda $\mathrm{Si}$ a la pregunta referido a la DAP será menor. Asimismo, la ecuación (5) solo permite estimar la diferencia $a=\left(a_{1}-a_{0}\right)$, el mismo que representa el cambio de utilidad por la mejora de la calidad de un bien o servicio (agua 
y saneamiento). Se induce entonces que el pago que dejaría indiferente al entrevistado, es decir $\Delta v$ $=0$, es igual al cambio en utilidad dividido por la utilidad marginal del ingreso $\beta$, tal como se muestra en la ecuación siguiente:

$0=a-\beta A_{t}$

$A_{t}=a / \beta$

La ecuación (6) representa la medida monetaria del cambio en el nivel de utilidad, este muestra la cantidad de dinero que está dispuesto a pagar (DAP) el entrevistado.

Pero como se observa en la ecuación (1), la función de utilidad del individuo no solo depende del vector de precios $p$ y el ingreso $y$, sino también de otras variables explicativas $q_{i}$ relacionadas con las características sociales y económicas que sirven para estimar la DAP, por ello, si incluimos mencionadas variables en la ecuación (6), la medida del cambio en el nivel de utilidad se expresaría como:

$$
\begin{aligned}
& A_{t}=\left(a_{0}+\sum_{i=1}^{k} a_{i} q_{i}\right) / \beta \\
& A_{t}=\frac{a q_{i}}{\beta}
\end{aligned}
$$

Donde $a$ `es la transpuesta del vector de parámetros, $q_{i}$ el conjunto de características socioeconómicas que no incluye el ingreso y $\beta$ es el coeficiente del precio.

El método de valoración contingente a lo largo de los años se ha convertido en una herramienta muy útil como apoyo en la evaluación de proyectos de provisión de servicios de agua, por lo que distintos autores han realizado este tipo de investigación para distintas ciudades que incluyen el estado de Onto en Nigeria (Akeju, Oladehinde, \& Abubakar, 2018), franja de Gaza (Al-Ghuraiz \& Enshassi, 2005), Karachi en Pakistán (Asim \& Lohano, 2015), este de Etiopia en África (Bogale \& Urgessa, 2012), Cusco en Perú (Guzmán, 2015), Songzi en China (Jianjun et al., 2016), Nsukka en Nigeria (Kanayo, Ezebuilo, \& Maurice, 2013), Jigjiga en Etiopia (Kebede \& Tariku, 2016), Lubombo y Lowveld en Swazilanda (Mvangeli, 2015), el Salvador (Perez \& Quintanilla, 2013), Mútale en Sudáfrica (Rananga \& Gumbo, 2015), Sucre en Bolivia (Saz, González, \& Guardiola, 2015), región de Pavlodar en Kazajistán (Tussupova, Berndtsson, Bramryd, \& Beisenova, 2015) y Vietnam (Van Minh, Nguyen-Viet, Thanh, \& Yang, 2012). Estos estudios también se realizaron para la ciudad de Puno en las que resaltan Tudela, Leos, \& Zavala, (2018) y Tudela (2017). A continuación, se presenta un breve resumen de los mismos.

Tabla 1

\begin{tabular}{|c|c|c|c|c|}
\hline Autor & Año & Objeto de estudio & Formato & Resultado \\
\hline $\begin{array}{l}\text { Akeju, Oladehinde y } \\
\text { Abubakar }\end{array}$ & 2018 & $\begin{array}{l}\text { Estiman la DAP por la mejora en el suministro del servicio de agua en Owo } \\
\text { estado de Ondo, Nigeria. }\end{array}$ & Referéndum & $\begin{array}{l}\mathrm{DAP}=1.617 \\
\text { nairas } / \text { mes }\end{array}$ \\
\hline $\begin{array}{l}\text { Al-Ghuraiz y } \\
\text { Enshassi }\end{array}$ & 2005 & Evalúan la DAP por el servicio de suministro de agua en la Franja de Gaza. & Referéndum & $\begin{array}{l}\text { DAP }=3.1 \\
\text { nuevos shekel } \\
\text { israeli } / \mathrm{m}^{3}\end{array}$ \\
\hline Asim y Lohano & 2015 & Estiman la DAP de los habitantes de Karachi, Pakistán. & Referéndum & $\begin{array}{l}\mathrm{DAP}=2.116 \\
\text { rupias } / \text { mes }\end{array}$ \\
\hline Bogale y Urgessa & 2012 & $\begin{array}{l}\text { Analizan la DAP por una mejor provisión de servicios de agua de los } \\
\text { hogares rurales del distrito de Haramaya, Etiopia. }\end{array}$ & Doble Limite & $\begin{array}{l}\mathrm{DAP}=1.17 \\
\text { dólares } / \text { mes }\end{array}$ \\
\hline Guzmán & 2015 & $\begin{array}{l}\text { Analiza el valor económico de mejoras en los servicios ambientales en el } \\
\text { contorno del rio Huatanay en el departamento de Cusco, Perú. }\end{array}$ & Doble Limite & $\begin{array}{l}\mathrm{DAP}=5.0 \\
\text { soles } / \mathrm{mes}\end{array}$ \\
\hline $\begin{array}{l}\text { Jianjun, Wenyu, } \\
\text { Ying, y Wang }\end{array}$ & 2016 & $\begin{array}{l}\text { Determina la DAP por la mejora en la calidad del agua y la confiabilidad del } \\
\text { suministro de los residentes locales en Songzi, China. }\end{array}$ & Referéndum & $\begin{array}{l}\mathrm{DAP}=16.71 \\
\text { yuanes } / \text { mes }\end{array}$ \\
\hline $\begin{array}{l}\text { Kanayo, Ezebuilo, y } \\
\text { Maurice }\end{array}$ & 2013 & $\begin{array}{l}\text { Analizan la DAP por los servicios de agua en el área de Nsukka, sureste de } \\
\text { Nigeria }\end{array}$ & Referéndum & $\begin{array}{l}\mathrm{DAP}=2.30 \\
\text { nairas } / \mathrm{mes}\end{array}$ \\
\hline Kebede y Tariku & 2016 & Determinan la DAP de los habitantes en la ciudad de Jigjiga, Etiopia & Referéndum & $\begin{array}{l}\text { DAP }=94 \\
\text { centavos } / 201 t\end{array}$ \\
\hline Mvangeli & 2015 & $\begin{array}{l}\text { Evalúa la DAP por la mejoría del servicio de agua en las regiones de } \\
\text { Lowveld y Lubombo, Swazilandia. }\end{array}$ & Doble Limite & $\begin{array}{l}\mathrm{DAP}=0.471 \\
\text { esuatinis } / \text { mes }\end{array}$ \\
\hline Perez y Quintanilla & 2013 & $\begin{array}{l}\text { Determinan la DAP por una mejora en la calidad de los servicios de agua y } \\
\text { saneamiento en las comunidades rurales de El Salvador. }\end{array}$ & Referéndum & $\begin{array}{l}\mathrm{DAP}=8.32 \\
\text { colones/mes }\end{array}$ \\
\hline Rananga y Gumbo & 2015 & $\begin{array}{l}\text { Evalúan la DAP por los servicios de agua potable en la localidad periurbana } \\
\text { de Tshilamba y la aldea rural de Tshlavulu de la Municipalidad local de } \\
\text { Mútale, Sudáfrica. }\end{array}$ & Referéndum & $\begin{array}{l}\text { DAP }=150 \\
\text { rand sud./6kl de } \\
\text { agua }\end{array}$ \\
\hline $\begin{array}{l}\text { Saz, González, y } \\
\text { Guardiola }\end{array}$ & 2015 & $\begin{array}{l}\text { Analizan la DAP por la mejora del abastecimiento urbano del servicio de } \\
\text { agua de los habitantes de Sucre, Bolivia. }\end{array}$ & Referéndum & $\begin{array}{l}\mathrm{DAP}=10.53 \\
\text { pesos bol/mes }\end{array}$ \\
\hline $\begin{array}{l}\text { Tudela, Leos, y } \\
\text { Zavala }\end{array}$ & 2018 & $\begin{array}{l}\text { Determina los beneficios económicos de la mejora integral en la provisión de } \\
\text { servicios de saneamiento básico en la ciudad de Puno, Perú. }\end{array}$ & Doble Limite & $\begin{array}{l}\text { DAP }=8.53 \\
\text { soles } / \mathrm{mes}\end{array}$ \\
\hline Tudela & 2017 & $\begin{array}{l}\text { Determina los beneficios económicos a generar por la ejecución del proyecto } \\
\text { de mejoramiento del sistema de tratamiento de aguas residuales de la ciudad } \\
\text { de Puno de la bahía interior del lago Titicaca (BILT), Perú. }\end{array}$ & Doble Limite & $\begin{array}{l}\mathrm{DAP}=4.38 \\
\text { soles } / \mathrm{mes}\end{array}$ \\
\hline $\begin{array}{l}\text { Tussupova, } \\
\text { Berndtsson, Bramryd, } \\
\text { y Beisenova }\end{array}$ & 2015 & $\begin{array}{l}\text { Investigan la DAP por la mejora de los servicios en el suministro del servicio } \\
\text { de agua para la región de Pavlodar, Kazajistán. }\end{array}$ & Referéndum & $\begin{array}{l}\mathrm{DAP}=150 \\
\text { tenges kaz/mes }\end{array}$ \\
\hline $\begin{array}{l}\text { Van Minh, Nguyen- } \\
\text { Viet, Thanh, y Yang }\end{array}$ & 2012 & $\begin{array}{l}\text { Evalúan la DAP por la mejora del servicio de saneamiento en la zona rural } \\
\text { del norte de Vietnam. }\end{array}$ & Referéndum & $\begin{array}{l}\mathrm{DAP}=7.80 \\
\text { dólares } / \text { mes }\end{array}$ \\
\hline
\end{tabular}

Revisión empírica de antecedentes literarios 


\section{METODOLOGÍA}

El alcance de la investigación es el centro poblado de Paxa, ubicado en el distrito de Tiquillaca, provincia de Puno, Perú. La investigación sigue un enfoque cuantitativo (Hernández, Fernández, \& Baptista, 2014), de carácter descriptivo, explicativo (Behar, 2008) y correlacional.

El tamaño de muestra se determinó en base a la información del INEI (2007) y a la técnica del muestreo aleatorio simple (MAS) determinándose así un tamaño de muestra de 112 hogares. La encuesta como instrumento de recopilación de información fue aplicada a los jefes de familia en dos sesiones (3 y 10) de diciembre del año 2017 con apoyo del personal de la municipalidad distrital de Tiquillaca. Para contrastar la validación del formato de la encuesta, se aplicó una encuesta piloto a 24 jefes de familia elegidos al azar, ello con fines de descartar respuestas negativas y grotescas (Jorgensen, Syme, Bishop, \& Nancarrow, 1999) e identificar los rangos mínimo y máximo de las tarifas a considerar en la encuesta final.

\section{Modelo referéndum de DAP}

Este formato consiste en presentarle al entrevistado dos alternativas de respuesta (si-no), para ello el individuo deberá expresar solo una respuesta. La probabilidad de obtener una respuesta afirmativa (si) a la pregunta de disponibilidad a pagar según Tudela \& Leos (2017) estaría dado por la ecuación:

$$
\operatorname{Pr}(s i)=\operatorname{Pr}\left[\Delta v>\varepsilon_{0}-\varepsilon_{1}\right]=F n
$$

Dado la naturaleza binaria de la variable dependiente, el análisis de regresión econométrica se realiza mediante un modelo logit o probit. Esta investigación uso el modelo Logit debido que admite mayor varianza en la distribución del término error (Tudela, 2007). La forma de representación del modelo logit se plantea como:

$$
\operatorname{Prob}(S i)=\frac{1}{1+\exp ^{-(a+\beta p)}}
$$

Según los autores, Tudela \& Leos (2017) el problema de estimación econométrica se resuelve a través del método de máxima verosimilitud dado por:

$$
L=\prod_{i=1}^{n}\left(1 F\left(\beta^{\prime} x_{i}\right)\right)^{1-y_{i}}\left(F\left(\beta^{\prime} x_{i}\right)\right)^{y_{i}}
$$

La función de logaritmo de verosimilitud (loglikelihood) es como la ecuación siguiente:

$$
L L=\sum_{i=1}^{n}\left[\left(1-y_{i}\right) \ln \left(1-F\left(\beta^{\prime} x_{i}\right)\right)+y_{i} \ln \left(F\left(\beta^{\prime} x_{i}\right)\right)\right]
$$

Dónde $y_{i}$ representa la variable dependiente binaria y $\beta^{\prime} x_{i}=\beta_{0}+\beta_{1} x_{1}+\ldots+\beta_{i} x_{i}$

\section{Modelo doble límite de DAP}

Para Tudela (2017), en el contexto de doble limite la pregunta inicial propuesta al individuo " $i$ " $\left(\beta_{i}\right)$ es repreguntada en función a la primera respuesta $\left(\beta_{i}^{d} o \beta_{i}^{u}\right)$, donde $\beta_{i}^{u}$ es el segundo precio propuesto después de una respuesta positiva al primero, $\beta_{i}^{d}$ es el segundo precio propuesto después de una respuesta negativa al primero. Según Hanemann et al. (1992) y Habb y McConell (2002) citados en Tudela \& Leos (2017), las respuestas en términos de probabilidad pueden expresarse como las ecuaciones siguientes:

$$
\begin{aligned}
& \operatorname{Pr}(s i, s i)=1-F\left(\beta^{\prime} x_{i}^{u}\right) \\
& \operatorname{Pr}(s i, n o)=F\left(\beta^{\prime} x_{i}^{u}\right)-F\left(\beta^{\prime} x_{i}\right) \\
& \operatorname{Pr}(n o, s i)=F\left(\beta^{\prime} x_{i}\right)-F\left(\beta^{\prime} x_{i}^{d}\right) \\
& \operatorname{Pr}(n o, n o)=F\left(\beta^{\prime} x_{i}^{d}\right)
\end{aligned}
$$

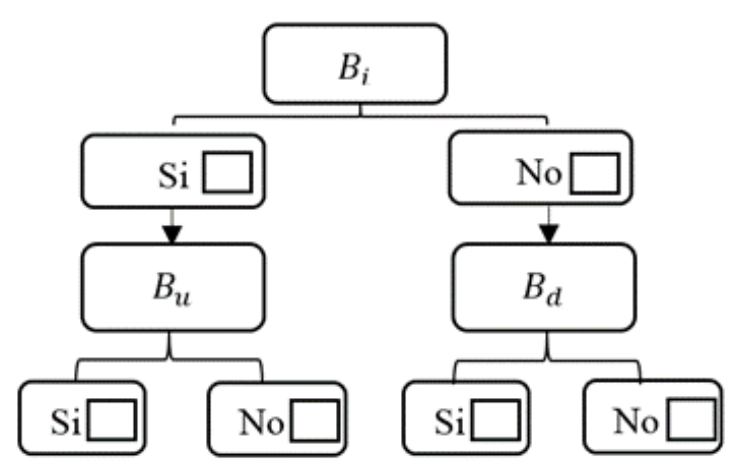

Figura 1. Ilustración grafica - formato doble limite. Fuente: Tudela (2017).

Elaboración: Propia. 
Donde el problema de estimación se resuelve a través del método de máxima verosimilitud (MV) con la función de densidad conjunta dado por:

$$
L=\prod_{i=1}^{n}\left(1-F\left(\beta^{\prime} x_{i}^{u}\right)\right)^{d_{i}^{s}}\left(F\left(\beta^{\prime} x_{i}^{u}\right)-F\left(\beta^{\prime} x_{i}\right)\right)^{d_{i}^{s}}\left(F\left(\beta^{\prime} x_{i}\right)-\left(F\left(\beta^{\prime} x_{i}^{d}\right)\right)^{d_{i}^{n n}}\right.
$$

La función de logaritmo de verosimilitud (log- likelihood), por ende presenta la siguiente forma:

$$
L L=\sum_{i=1}^{n}\left\{\begin{array}{r}
d_{i}^{s s} \ln \left[1-\frac{1}{\left.1+\exp ^{-\left(\beta^{\prime} x_{i}^{u}\right)}\right]}\right]+d_{i}^{s n} \ln \left[\frac{1}{1+\exp ^{-\left(\beta^{\prime} x_{i}^{d}\right)}}-\frac{1}{1+\exp ^{-\left(\beta^{\prime} x_{i}\right)}}\right] \\
+d_{i}^{n s} \ln \left[\frac{1}{1+\exp ^{-\left(\beta^{\prime} x_{i}\right)}}-\frac{1}{1+\exp ^{-\left(\beta^{\prime} x_{i}^{d}\right)}}\right]+d_{i}^{n n} \ln \left[1-\frac{1}{1+\exp -\left(\beta^{\prime} x_{i}^{d}\right)}\right]
\end{array}\right\}
$$

Dónde: $d_{i}^{s s}, d_{i}^{s n}, d_{i}^{n s}, d_{i}^{n n}$ son las variables binarias creadas a partir de las respuestas si-sí, si-no, nosi y no-no, los mismos que toman el valor de 1 cuando la respuesta del entrevistado se encuentra en alguna de estas alternativas, y 0 si es lo contrario.

\section{Relación capacidad de pago y DAP a través del análisis de correlación de Pearson}

El coeficiente de Pearson mide la fuerza de la relación lineal entre los valores cuantitativos apareados $x$ y $y$ en una muestra (Triola, 2009). Según Martínez (2012) cuando la muestra (n) es pequeña, se emplea la distribución " $t$ " y " $z$ " para $n>30$; se maneja dos hipótesis a comprobar:

$H_{0}: r=0$ (No hay correlación $)$

$H_{\mathrm{a}}: r>0($ Si hay correlación $)$

\section{Metodología Capacidad de Pago según el Sistema Nacional de Programación Multianual y Gestión de Inversiones}

Invierte.pe, nuevo sistema de inversión pública en el Perú, nace con la finalidad de orientar el uso de los recursos públicos destinados a la inversión para la efectiva prestación de servicios y la provisión de infraestructura necesaria para el desarrollo del país. Para ello el Ministerio de Vivienda, Comunicación y Saneamiento elaboró la "Ficha Técnica para la Formulación de Proyectos de Saneamiento en el Ámbito Rural". En el numeral 9.2 se hace mención a la capacidad de pago, cuya fórmula para el caso de dotación de servicio de agua potable y alcantarillado es:
$C P=I n g$ Promedios $(S /$ f am / mes $) * 0.05$

\section{RESULTADOS Y DISCUSIÓN}

En la encuesta de valoración contingente tipo referéndum el análisis de la DAP para la sostenibilidad del proyecto, la tabla 2 revela que del $100 \%$ de encuestados, $54.46 \%$ tiene voluntad de pago, mientras que el $45.54 \%$ revelaron no estar dispuestos a pagar para la sostenibilidad del proyecto. La tabla 3 muestra las respuestas para el formato doble límite, donde $42.86 \%$ de encuestados respondieron de manera positiva a la DAP (posturas Si - Si y No - Si), mientras que el $57.14 \%$ respondieron de manera negativa a la DAP (posturas Si - No y No - No). Dichos resultados difieren de manera significativa a lo obtenido en el formato referéndum, donde existe mayor porcentaje de respuestas positivas a la pregunta referente a la DAP.

Tabla 2

Respuestas a la pregunta referente a la DAP - referéndum

\begin{tabular}{cccc}
\hline Rango de & Número de & \multicolumn{2}{c}{ Respuestas afirmativas } \\
\cline { 3 - 4 } tarifas $(\mathrm{S} /)$. & encuestas & Frecuencia & $\%$ \\
\hline 1.50 & 14 & 13 & 92.86 \\
2.00 & 14 & 11 & 78.57 \\
2.50 & 14 & 10 & 71.43 \\
3.00 & 14 & 4 & 28.57 \\
3.50 & 14 & 7 & 50.00 \\
4.00 & 14 & 4 & 28.57 \\
4.50 & 14 & 4 & 28.57 \\
5.00 & 14 & 8 & 57.14 \\
\hline Total & 112 & 61 & 54.46 \\
\hline
\end{tabular}

Fuente: Encuestas realizadas los días 3 y 10 de diciembre del 2017.

Elaboración: Propia 
Tabla 3

Respuestas a preguntas iterativas - doble límite

\begin{tabular}{ccccccc}
\hline Rango de & \multicolumn{5}{c}{ Respuestas } \\
\cline { 2 - 7 } tarifas (S/.) & Si & No & Si - Si & Si - No No - Si No - No \\
\hline 1 & & & & & 0 & 1 \\
1.5 & 13 & 1 & & & 0 & 3 \\
2 & 11 & 3 & 4 & 9 & 2 & 2 \\
2.5 & 10 & 4 & 6 & 5 & 3 & 7 \\
3 & 4 & 10 & 6 & 4 & 1 & 6 \\
3.5 & 7 & 7 & 3 & 1 & 6 & 4 \\
4 & 4 & 10 & 5 & 2 & 4 & 6 \\
4.5 & 4 & 10 & 2 & 2 & 3 & 3 \\
5 & 8 & 6 & 2 & 2 & & \\
5.5 & & & 1 & 7 & & \\
\hline Total & 61 & 51 & 29 & 32 & 19 & 32 \\
\hline
\end{tabular}

Fuente: Encuestas realizadas los días 3 y 10 de diciembre del 2017. Elaboración: Propia.

\section{Análisis Econométrico}

Los resultados de las regresiones econométricas tipo referéndum y doble límite se aprecian en la tabla 4. En el formato referéndum se selecciona el Logit 2, bajo los siguientes criterios de decisión:

Prueba de Akaike (AIC): propone una corrección a los estadísticos log-likelihood y LR-statistic por el número de parámetros del modelo (coeficientes de regresión).

Criterio de selección: Valor de $A I C$ menor.

$$
A I C_{\text {logit } 2}<A I C_{\text {logit } 1}
$$

Prueba de Schwarz (SC): Prueba que permite comparar la bondad de ajuste entre dos modelos.

Criterio de selección: Valor de $S C$ menor.

$$
S C_{\text {logit } 2}<S C_{\text {logit1 }}
$$

Test individual mediante la prueba " $z$ ": el test de significatividad individual sirve para juzgar si una determinada variable independiente debe ser incluida o no en la especificación del modelo. Para el trabajo de investigación se trabajó a un nivel de significancia al $1,5 \mathrm{y}$ $10 \%$.

Luego de seleccionar el modelo Logit 2, se precedió a realizar un análisis comparativo con el modelo doble límite, de ambas regresiones, se seleccionó este último, dado los siguientes criterios de decisión:

Razón de Verisimilitud $(R V)$ : el estadístico $R V$ es análogo a la prueba $\mathrm{F}$ de un modelo convencional, cuya estimación se realiza haciendo uso de la siguiente formula $L R=$ $-2\left[L n L_{r}-L n L\right]$, donde $L n L_{r}$ es la función de verosimilitud logarítmica evaluada en el estimador restringido y $L n L$ es la función de verosimilitud logarítmica no restringida. Dicho estadístico se contrasta con los valores críticos de una distribución chi-cuadrada.

Criterio de selección: Valor de razón de verosimilitud $R V$ mayor.

$$
L R_{\text {doble limite }}>L R_{\text {logit } 2}
$$

Asimismo, los resultados del modelo doble límite muestran que los signos de los coeficientes de las variables son los esperados, es decir:

$\checkmark$ El signo del coeficiente de la variable PHI (precio) es negativo. Esto quiere decir que, ante un aumento en la tarifa ofrecida para la sostenibilidad del proyecto, la probabilidad de obtener una respuesta positiva por parte del encuestado a la pregunta de la DAP es menor.

$\checkmark$ El signo del coeficiente de la variable ING (ingreso) es positivo, indica que a mayor nivel de ingreso familiar, incrementa la probabilidad de obtener una respuesta positiva a la pregunta de la DAP.

$\checkmark$ El signo del coeficiente de la variable EDAD (edad) es negativo, es decir, a mayor edad del encuestado, la probabilidad de obtener una respuesta positiva a la pregunta de la DAP es menor.

$\checkmark$ El signo del coeficiente de la variable EDUC (educación) es positivo, el mismo que indica que a mayor nivel educativo (a mayor años de estudio), aumenta la probabilidad de que el encuestado responda de manera positiva a la pregunta de la DAP. 
De igual forma, los estadísticos " $\mathrm{z}$ ” indican que las variables independientes son significativas al $5 \%$. La significancia conjunta es muy alta en términos del estadístico $\mathrm{LR}=296.80$, el valor crítico de un chi cuadrado al 5\% de significancia con 5 grados de libertad es 14.07, por lo que se rechaza la hipótesis conjunta de que los coeficientes de todas las variables independientes sean 0 .

Tabla 4

Estimaciones econométricas modelo logit - referéndum

\begin{tabular}{|c|c|c|c|}
\hline \multirow{3}{*}{ Variables } & \multicolumn{2}{|c|}{ Modelo Referéndum } & Modelo Doble limite \\
\hline & \multicolumn{3}{|c|}{ Coeficientes y Nivel de Significancia } \\
\hline & Modelo logit 1 & Modelo logit 2 & Doble limite \\
\hline \multirow{2}{*}{ Constante } & 13.59331 & 6.79370 & 4.84835 \\
\hline & $(1.878)^{*}$ & $(1.234)$ & $(3.429)^{* *}$ \\
\hline \multirow{2}{*}{ PHI } & -6.21651 & -4.00254 & -1.36563 \\
\hline & $(-3.025)^{* *}$ & $(-3.566) * *$ & $(-6.928)^{* * *}$ \\
\hline \multirow{2}{*}{ ING } & 10.43477 & 7.46273 & 0.94310 \\
\hline & $(3.190)^{* *}$ & $(3.599)^{* *}$ & $(3.158)^{* *}$ \\
\hline \multirow{2}{*}{ EDAD } & -0.31358 & -0.18487 & -0.07289 \\
\hline & $(-2.094)^{* *}$ & $(-1.950)^{*}$ & $(-3.231)^{* *}$ \\
\hline \multirow{2}{*}{ EDUC } & 0.51408 & 0.40271 & 0.10690 \\
\hline & $(2.347)^{* *}$ & $(2.154)^{* *}$ & $(2.082)^{* *}$ \\
\hline \multirow{2}{*}{$\mathrm{TH}$} & 0.66999 & & \\
\hline & $(0.750)$ & & \\
\hline \multirow{2}{*}{ DIST } & -0.00828 & & \\
\hline & $(-0.933)$ & & \\
\hline \multirow{2}{*}{ TIEMP } & 0.03004 & & \\
\hline & $(0.164)$ & & \\
\hline \multirow{2}{*}{ ENF } & 2.64766 & & \\
\hline & $(1.160)$ & & \\
\hline Logaritmo de verosimilitud & -9.15926 & -12.34500 & 148.4016 \\
\hline Razón de verosimilitud & 136.05 & 129.6809 & 296.80 \\
\hline Akaike I.C. & 0.32427 & 0.30973 & \\
\hline Schwarz I.C. & 60.78503 & 48.28249 & \\
\hline
\end{tabular}

Los números entre paréntesis representan los $\mathrm{z}-$ estadísticos; $* * *$ indica significancia a un nivel del $1 \%$, ** al $5 \%$ y $*$ al $10 \%$.

Fuente: Encuestas realizadas los días 3 y 10 de diciembre del 2017.

Elaboración: Propia a partir de los resultados del software N - Logit.

\section{Relación capacidad de pago y DAP}

En la tabla 5, se observa una relación directa entre la capacidad de pago y la DAP, asimismo en la figura 2 se puede ver que a mayor capacidad de pago mayor será la DAP del encuestado. Este resultado apoya a lo obtenido por Tudela (2017), quien elabora un índice de capacidad de pago (ICP) a través del análisis de componentes principales, cuyo resultado muestra una relación directa entre las variables DAP e ICP.
Tabla 5

Relación capacidad de pago y DAP

\begin{tabular}{ccc}
\hline & DAP & CP \\
\hline DAP & 1 & \\
CP & $0.847 * *$ & 1 \\
\hline$* *$ La correlación es significativa al $1 \%$.
\end{tabular}

Fuente: Encuestas realizadas los días 3 y 10 de diciembre del 2017.

Elaboración: Propia a partir de los resultados del software SPSS. 


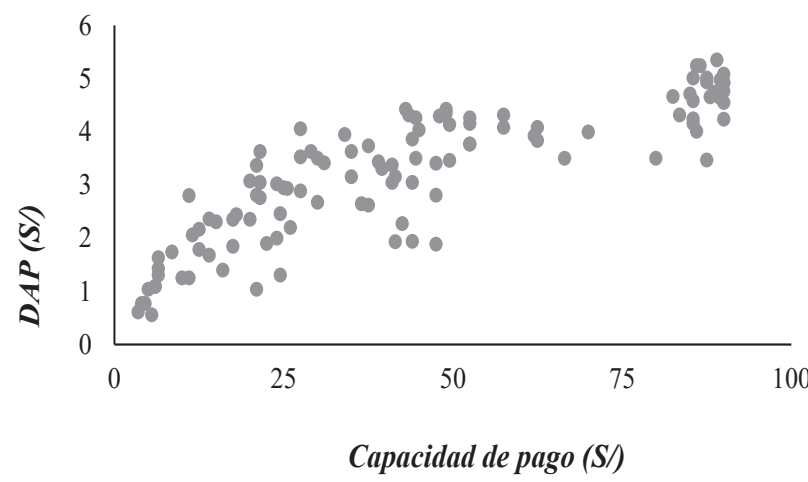

Figura 2. Relación capacidad de pago y DAP

Fuente: Encuestas realizadas los días 3 y 10 de diciembre del 2017.

Elaboración: Propia a partir de los resultados del software SPSS.

\section{Análisis de la DAP media}

Luego del análisis y validación de los resultados econométricos, se estimó la DAP media cuyos valores resultaron $\mathrm{S} / 3.85$ fam/mes y $\mathrm{S} / 3.22$ fam/mes en el modelo referéndum y doble límite respectivamente, tal como se muestra en la tabla 6. Asimismo, al comparar los resultados de las estimaciones econométricas obtenidas por el modelo referéndum en comparación al modelo doble límite (Tabla 4), se pudo apreciar que el nivel de significancia de los parámetros en este último se ajusta de un $10 \%$ a un $5 \%$, lo que hace una mejor aproximación a la verdadera DAP de los encuestados, reflejando una sobrestimación del valor de la DAP en el primer modelo. Cabe resaltar que el valor de la DAP puede diferir debido a las diferencias socioeconómicas $\mathrm{y}$, demográficas en distintas regiones del mundo.

\section{Tabla 6}

Resultados de la DAP media modelo referéndum $y$ doble límite

\begin{tabular}{lcc}
\hline Variable & Media & $\begin{array}{c}\text { Desviación } \\
\text { estándar }\end{array}$ \\
\hline DAP $_{\text {ref }}$ & 3.85 & 2.09 \\
DAP $_{\mathrm{dl}}$ & 3.22 & 1.24 \\
\hline
\end{tabular}

Fuente: Encuestas realizadas los días 3 y 10 de diciembre del 2017.

Elaboración: Propia a partir de los resultados del software N-Logit.

\section{CONCLUSIONES}

Los factores socioeconómicos que inciden en la DAP de las familias del centro poblado de Paxa son: el precio hipotético (PHI), el ingreso familiar mensual (ING), la edad (EDAD) y la educación (EDUC) del entrevistado, es necesario entonces que las autoridades velen y se comprometan de manera más coactiva en dichos factores para el mejoramiento del capital humano, así como del bienestar social.

La relación entre la capacidad de pago y la disponibilidad a pagar de las familias del centro poblado de Paxa, según el análisis de correlación de Pearson, resulto ser directa y positiva.

La disponibilidad a pagar (DAP) media de las familias del centro poblado de Paxa para la sostenibilidad del proyecto de inversión según el formato referéndum es de S/. 3.85 mes/fam.; y S/. $3.22 \mathrm{mes} / \mathrm{fam}$ en el formato doble limite, asimismo, luego del análisis de ambos formatos, el modelo doble limite es el que presentó mayor respaldo teórico, es por ello la selección del valor de su DAP como la verdadera DAP media de las familias del centro poblado de Paxa.

\section{CONFLICTOS DE INTERÉS}

Los autores declaran no tener conflictos de interés.

\section{REFERENCIAS BIBLIOGRÁFICAS}

Akeju, T., Oladehinde, G., \& Abubakar, K. (2018). An analysis of willingness to pay (WTP) for improved water supply in Owo local government, Ondo State, Nigeria. Asian Research Journal of Arts \& Social Sciences, 5(3), 1-15. https://doi.org/10.9734/ ARJASS/2018/39282

Al-Ghuraiz, Y., \& Enshassi, A. (2005). Ability and willingness to pay for water supply service in the Gaza Strip. Building and Environment, 40, 1093-1102. https://doi.org/10.1016/j. buildenv.2004.09.019 
Asim, S., \& Lohano, H. (2015). Households' willingness to pay for improved tap water services in Karachi, Pakistan. Pakistan Development Review, 54, 507-526. https:// doi.org/10.30541/v54i4I-IIpp.507-526

Behar, D. (2008). Introducción a la metodología de la investigación. Shalom (Vol. 1). Shalom. https://doi.org/10.1017/ CBO9781107415324.004

Bogale, A., \& Urgessa, B. (2012). Households' willingness to pay for improved rural water service provision: application of contingent valuation method in eastern Ethiopia. J Hum Ecol, 38(2), 145-154.

Gutierrez, S. (2015). Disponibilidad de pago para la sostenibilidad del servicio de agua potable en el C.P. Sucasco, Almozanche y localidad de Coata 2014. Universidad Nacional del Altiplano.

Guzmán, E. (2015). Valoración económica de mejoras en los servicios ambientales en el contorno del río Huatanay, Cusco- Perú. Consorcio de Investigación Económica y Social, 1-67. Retrieved from http://www.cies. org.pe/sites/default/files/investigaciones/a1 t2a_pbcus_3er_informe final_31.08.2015. pdf

Hernández, R., Fernández, C., \& Baptista, P. (2014). Metodología de la investigación. Mc Graw Hill (6ta ed., Vol. 53). Mexico D.F. https:// doi.org/10.1017/CBO9781107415324.004

INEI. (2007). Instituto Nacional de Estadística e Informática, Censos Nacionales XI de Poblacion y VI de Vivienda 2007.

INEI. (2018). Perú: formas de acceso a agua y saneamiento básico. Perú. Retrieved from https://www.inei.gob.pe/media/ MenuRecursivo/boletines/boletin agua_y saneamiento.pdf

Jianjun, J., Wenyu, W., Ying, F., \& Wang, X. (2016). Measuring the willingness to pay for drinking water quality improvements: results of a contingent valuation survey in Songzi, China. Journal of Water and Health, 14(3), 504-512. https://doi.org/10.2166/ wh.2016.247

Jorgensen, B., Syme, G., Bishop, B., \& Nancarrow,
B. (1999). Protest responses in contingent valuation. Environmental and Resource Economics, 14(1), 131-150. Retrieved from https://doi.org/10.1023/A:1008372522243

Kanayo, O., Ezebuilo, U., \& Maurice, O. (2013). Estimating the willingness to pay for water services in Nsukka area of south-eastern Nigeria using contingent valuation method (CVM): implications for sustainable development. Journal of Human Ecology, 41(2), 93-106. Retrieved from https://doi.or g/10.1080/09709274.2013.11906556

Kebede, S., \& Tariku, L. (2016). Households' willingness to pay for improved water supply: application of the contingent valuation method; evidence from Jigjiga town, Ethiopia. The Romanian Economic Journal, 19(62), 191-204. Retrieved from http://www.rejournal.eu/sites/rejournal. versatech.ro/files/articole/2017-01-03/3411/ yhundie.pdf

Martínez, C. (2012). Estadística y muestreo (13 ed.). Bogotá: Ecoe Ediciones.

Mendieta, C. (2005). Manual de valoración económica de bienes no mercadeables. CEDE. https://doi.org/10.2139/ssrn.1590558

Mvangeli, N. (2015). Households' water use demand and willingness to pay for improved water services: a case study of semi-urban areas in the Lubombo and Lowveld regions of Swaziland. Collaborative Masters Program in Agricultural and Applied Economics. Retrieved from https://ideas.repec.org/p/ags/ cmpart/243464.html

OMS. (2017). Agua potable. Retrieved from http:// www.who.int/mediacentre/factsheets/fs391/es/

OMS, \& UNICEF. (2017). Progress on drinking water, sanitation and hygiene 2017 update and SDG baselines. Retrieved from https:// www.unicef.org/lac/JMP-2017-report(1).pdf

Parra, A., Vargas, V., \& Castellar, C. (2005). Metodología estadística para estudios de disponibilidad a pagar (DAP), con aplicación en un proyecto de abastecimiento de agua. ResearchGate, (January). Retrieved from https://www.researchgate.net/publication/ 23743005 
Perez, F., \& Quintanilla, C. (2013). Estimating willingness to pay and financial feasibility in small water projects in El Salvador. Journal of Business Research, 66(10), 1750-1758. https://doi.org/10.1016/j.jbusres.2013.01.014 Rananga, H. . T., \& Gumbo, J. R. (2015). Willingness to pay for water services in two communities of Mutale local municipality, South Africa : a case study. Journal of Human Ecology, 49(3), 231-243. https://doi.org/10.1 $\underline{080 / 09709274.2015 .11906841}$

Saz, S., González, F., \& Guardiola, J. (2015). Willingness to pay to improve urban water supply: the case of Sucre , Bolivia. Water Policy, 17(1), 112-125. https://doi. org/10.2166/wp.2014.195

Triola, M. (2009). Estadística. (P. Educación, Ed.) (10 ed.). México.

Tudela, J. (2007). Estimación de la disponibilidad a pagar de los habitantes de la ciudad de Puno por el tratamiento de aguas servidas. CIES: Economía Y Sociedad, 68, 73-83. Retrieved from http://cies.org.pe/es/content/ estimacion-de-la-disponibilidad-pagar-delos-habitantes-de-la-ciudad-de-puno-por-el

Tudela, J. (2017). Estimación de beneficios económicos por el mejoramiento del sistema de tratamiento de aguas residuales en la ciudad de Puno (Perú). Desarrollo Y Sociedad, (79), 189-237. https://doi.org/10.13043/DYS.79.6

Tudela, J. W., \& Leos, J. A. (2017). Herramientas metodológicas para aplicaciones del método de valoración contingente (1 ra ed.). Mexico: Universidad Autónoma de Chapingo. Retrieved from https:// dialnet.unirioja.es/servlet/articulo? codigo $=6423191$

Tudela, W., \& Leos, J. (2017). Herramientas metodológicas para aplicaciones del método de valoración contingente (1a ed.).

Tudela, W., Leos, J., \& Zavala, M. (2018). Estimación de beneficios económicos por mejoras en los servicios de saneamiento básico mediante valoración contingente. Agrociencia, 52(3), 467-481. Retrieved from https://dialnet.unirioja.es/servlet/ articulo? codigo $=6423191$

Tussupova, K., Berndtsson, R., Bramryd, T., \& Beisenova, R. (2015). Investigating willingness to pay to improve water supply services: application of contingent valuation method. Water, 3024-3039. https://doi. org/10.3390/w7063024

UN-Water. (2016). Seguimiento en materia de agua y saneamiento en la agenda 2030 para el desarrollo sostenible. Retrieved from http:// www.unwater.org/app/uploads/2016/05/ Seguimiento-en-Materia-de-Agua-ySaneamiento-Una-introduccion_2016-06-01. pdf

UNICEF. (2007). Agua, saneamiento, higiene e infancia. Retrieved from https:// www.google.com.pe/url? sa $=$ t\&rct $=\mathrm{j} \& \mathrm{q}=\&$ es rc $=\mathrm{s} \& \mathrm{~s}$ ource $=\mathrm{web} \& \mathrm{~cd}=$ $15 \& \mathrm{cad}=\mathrm{rja} \& u a c t=8 \& \mathrm{ved}=0 \mathrm{ahUKEwiMz} 7$ cqL3XAhUGOiYKHSoMCasQFghp MA4\&url=https $\% 3 \mathrm{~A} \% 2 \mathrm{~F} \% 2 \mathrm{Fwww}$.unicef. org\%2Flac\%2FtemaspolpulAgua esp(1). pdf\&usg=AOvVaw $3 \mathrm{f} 3 \mathrm{zkEEPQUBm}$ TGlxQbAfgL

Van Minh, H., Nguyen-Viet, H., Thanh, N. H., \& Yang, J. C. (2012). Assessing willingness to pay for improved sanitation in rural Vietnam. Environmental Health and Preventive Medicine, 18(4). https://doi.org/10.1007/ s12199-012-0317-3

Vásquez, F., Cerda, A., \& Orrego, S. (2007). Valoración económica del ambiente (1a. ed.). Buenos Aires: Thomson.

Wang, H., Shi, Y., Kim, Y., \& Kamata, T. (2013). Valuing water quality improvement in China: a case study of Lake Puzhehei in Yunnan Province. Ecological Economics, 94, 56-65. $\quad$ https://doi.org/10.1016/j. ecolecon.2013.07.006 